\title{
Enabling a Comprehensive Teaching Strategy: Video Lectures
}

\author{
H. David Brecht and Suzanne M. Ogilby \\ California State University, Sacramento, California, USA
}

\author{
brecht@csus.edu; ogilbysm@csus.edu \\ Executive Summary
}

This study empirically tests the feasibility and effectiveness of video lectures as a form of video instruction that enables a comprehensive teaching strategy used throughout a traditional classroom course. It examines student use patterns and the videos' effects on student learning, using qualitative and nonparametric statistical analyses of survey responses and student grades. Groups of student course grades are compared with and without video lectures available.

Video lectures are CD and web viewable files that present lecture materials and narrative instruction from a course's instructor. They are used as additions to classroom lectures and are not recordings of classroom lectures. In these lectures, the instructor uses Microsoft Office content files, narrative instruction, and screen writing with the keyboard and mouse pointer to deliver the lecture.

Video lectures serve major strategic purposes. First, they give additional teaching time to students who cannot fully understand the course material through the classroom lectures and support materials such as the textbook. Students can view and study the instructor's lectures as often as they wish until they understand material. This study resource is particularly important in teaching a broad spectrum of students.

Second, video lectures allow classroom coverage of more complex and challenging subject material that is more interesting to many students. Students who are overwhelmed by these information-packed classroom lectures have the video lectures to study, which cover both the basics and the more advanced material. Moreover, some of the time freed in classroom lectures can be converted to problem-solving lab days that focus on active learning.

On these lab days, students are given complex and topic-comprehensive problems to solve. These problems aggressively challenge and move forward students' understanding and skill in working with the course material. Because video lectures are available as a support teaching resource, the labs can be used to involve students in solving problems that integrate multiple threads of a topic.

Survey results indicate that many students accept and use video lectures as contemplated by this

Material published as part of this publication, either on-line or in print, is copyrighted by the Informing Science Institute. Permission to make digital or paper copy of part or all of these works for personal or classroom use is granted without fee provided that the copies are not made or distributed for profit or commercial advantage AND that copies 1) bear this notice in full and 2) give the full citation on the first page. It is permissible to abstract these works so long as credit is given. To copy in all other cases or to republish or to post on a server or to redistribute to lists requires specific permission and payment of a fee. Contact Publisher@InformingScience.org to request redistribution permission. teaching strategy. The extent to which students use the videos is expected to be associated with more than the need for additional teaching time since alternative study resources are always available. Video use frequency and patterns are expected to be at least in part related to each student's learning style preferences. The paper identifies some types 
of learning style preferences to which video lectures appeal.

A statistically significant $68.5 \%$ of students using the videos said they helped understand course information and prepare for midterm exams. A statistically significant $72.2 \%$ said the videos helped do homework and prepare for weekly exams. Moreover, a statistically significant $63 \%$ of students said they provide tutoring help. These use patterns indicate broad acceptance of the computer-based instruction in video lectures, delivered by the course instructor, as a supplementary resource in a traditional course. They indicate that video lectures appeal to many students for a variety of purposes, and are effective for learning.

Regarding course grades, survey responses indicate that the video lectures helped $20.4 \%$ of students pass the course. Observing grade data, $24.2 \%$ of students without the videos failed the course. This rate dropped to $6.8 \%$ for the sample group with the videos available - a substantial improvement. Evaluating video lectures as a study resource for comprehensive final exams, they provide an entire semester's lectures delivered by the instructor. Final exam data show that the availability of video lectures improves final exam grades for all grade ranges.

Keywords: IT and innovative pedagogy, computer video lectures, video instruction, IT and teaching strategy, IT and learning style preferences

\section{Introduction}

This research contributes to the resolution of two problems that occur in university-level education. One is how to help otherwise failing students or students uninterested in studying using traditional classroom lecture and text reading methods become academically successful. (At the university level, students' inability to learn or succeed in passing classes becomes a problem of retention.) A second is an aspect of "teaching to or below the mean" - how to avoid dilution of the curriculum and increase the complexity and amount of subject material covered in a course when a nontrivial segment of the class has difficulty learning the basics. Addressing these problems is particularly important in a university with a mission of teaching a broad spectrum of students.

This study applies video lectures (a specific application of the broader technology "video instruction") as a way to leverage information technology (IT), not simply as a tool for recording and distributing information, but as a way to enable a comprehensive teaching strategy used throughout a course - a strategy that addresses these problems. Many different computer applications provide information to students and instruction - applications ranging from online lecture notes to limited topic, off-the-shelf instructional videos or simulations or to instructional videos that have been developed by educators as pedagogical methods used for their individual courses. As used in this study, video lectures are substantively different, delivering the entire topic content taught in a course's classroom lectures as portable lectures that students can rehear and study. Their content and pattern of topic development are specific and inclusive to what an individual instructor covers in the classroom.

More important than being portable lectures to support initial learning or for review before an exam, video lectures enable a comprehensive instructional strategy that allows greater depth of coverage, with more classroom time spent on complex material that is interesting to many students and less time spent on basic explanations. Additionally, they support converting a large percentage of class time from classroom lectures to problem-solving labs that emphasize active learning. The video lectures are available to support students needing additional teaching time, whether on the basic or more complex subject material.

Besides presenting the strategic design for this use of video lectures, this study provides initial empirical evidence of outcomes from their use. Outcomes include student perceptions of the videos' value, how they used the videos, and the videos' effects on student learning. This evidence 
adds to the literature, where the education literature has noted that the learning effectiveness of many IT applications is relatively unknown (Maki \& Maki, 2000).

\section{Contribution to the Literature}

Elements of the current study are part of other studies but are considered in different ways. For example, Hornik reports the effect on student retention, course grade performance, and learningprocess satisfaction of using IT-mediated learning with no face-to-face communication, e.g., online education (Hornik, Saunders, Li, Moskal, \& Dzuiban, 2008). This research finds higher success when IT is used for instruction in introductory courses with a less well-developed structure of concepts and techniques than when this structure is highly developed.

Alternatively, the present study evaluates the same outcomes but uses the more specific video lecture technology to re-lecture material that is presented in classroom lectures. The videos also support active-learning activities by providing complete lectures that can be reviewed for their coverage of concepts and methods occurring in difficult problems worked on in the labs. An additional study difference is that in the current study comparative success is found from using video lectures when the course is introductory and has a highly developed concept/methods structure. Sample groups are compared with and without video lectures available.

Keengwe addresses the integration of technology into classroom education (Keengwe, 2007). By reference to the literature (Cuban, 2001), Keengwe states, "Few faculty have demonstrated effective and efficient computer technology integration use in their classrooms" (Keengwe, p. 2). Additionally, he says, "Few studies are available on students' perceptions of computer technology use." The current study adds to the literature empirical evidence of video lectures' integration as a study resource in traditional classrooms and students' acceptance and patterns of use of this resource.

Sarker presents an overview of the literature on online education and states that there have been few empirical studies to date (Sarker \& Nicholson, 2005). The studies referenced consider webbased versus traditional classroom outcomes; the effects of active-learning techniques used in a virtual environment versus those obtained in traditional classrooms; and effects for online, traditional, and mixed learning environments. No significant outcome differences were found between the applications and environments compared in any of these studies. The current study is solely of an in-residence, classroom environment that mixes traditional instruction methods with activelearning and video lectures. Successful outcomes were found for the sample group using the videos.

As suggested by most of the above studies, the papers they reference, and a general review of internet links, there are many studies related to the online learning environment. Within this environment, there are studies that apply or examine aspects of video instruction. The current study is different in that it addresses the strategic use of video instruction in support of a traditionalclassroom learning environment. This strategic use connects video lectures as an enabling pedagogy to a greater depth of study in a course and a conversion of classroom time from lectures to active learning labs.

Whatley and Ahmad (2007) address the development of relatively short videos that summarize key material previously presented, not in an online course, but in the classroom or lecture hall. Their intent is to support student review of basic concepts, for either initial learning or assessments review. Differently in the current study, video lectures are full-length instructional lectures of all course materials. Their purposes are the same, which are to support initial learning and exam review (assessment). 
For the technology, Whatley and Ahmad use digital video cameras to record either live lectures that were edited into short summaries or in-office summary lectures. As discussed in the next section, the current study's video lectures are complete lectures that parallel the full content presented in classroom lectures. They are created solely on a personal computer, using content files and narrative audio - both encoded into a video with easy to use software and without editing.

\section{Study Design \& Analysis Methodology}

The video application offered in this study, and supported by the empirical evidence collected, is an innovative contribution to the literature. The basis of the application is to improve student learning and retention, support the strengthening of course content and subject complexity, and support the use of classroom time to engage students in active learning. The evidence collected is an initial effort to evaluate student acceptance and use of video lectures and the videos' effects on grades/learning. The course is introductory financial accounting and is not advertised as computer enhanced. Thus, the students did not self-select a computer-enhanced learning environment. They represent a random mix of student technical capabilities and orientation, academic interests, and aptitudes that might be found in any business school introductory, required course.

The instrument for gaining information about acceptance and use was a survey given at the end of the course, but before the final exam, to the sample group of students that had video lectures available. To get unbiased responses, the survey was anonymous. This effect is validated in that $27 \%$ of the responding students said they did not use the videos at all and several important survey questions did not receive statistically significant affirmative responses. These responses indicate that students critically evaluated the video lectures and responded without a bias to please the instructor.

Also, especially regarding reported student use of the videos, students were not forced to use them to access or learn the course material. Alternative study resources were always available (classroom lectures, instructional PowerPoint files, homework assignments and solutions, lab practice problems and solutions, and the textbook). Thus, use of the videos, topic by topic and week by week, was always voluntary and hidden from the instructor's view. This removed a potential bias from student responses to the survey.

A secondary but important focus of the study is the effect of video lectures on student learning as measured by survey responses and course grades. Of especial interest is the effect of using videos for students in danger of failing the course. The direct evidence available for this part of the study is limited to the student survey responses, where anonymous students self-identify using the videos and state whether or not they helped pass the course. Individual students could not be identified as using the video lectures and then traced to their actual grade performance. However, indirect evidence is examined of whether video lecture availability is associated with higher course grades. The two sets of evidence are compared for consistency and implied validity.

Qualitative and nonparametric statistical analyses are used to examine the survey and course grade evidence. The qualitative analysis evaluates evidence for reasonableness and consistency in view of other available evidence, possible underlying explanations for the result, and expectations. The statistical analyses address whether or not a survey response or a course grade outcome is random. Nonparametric analysis is used for three reasons. First, yes/no survey responses are binomial, although the binomial tends toward the normal distribution with larger sample sizes. Second, the effects of video use on a course grade distribution are unknown, making a distribution-based norm problematic to use. In fact, it is expected that video use will change the shape of the grade distribution, but it is not known what this distribution will look like or what should be a comparative norm. And third, the binomial distribution is well suited for rankings. Grades 
grouped by intervals are essentially data ranked into the performance classifications of $\mathrm{F}-\mathrm{A}$, from worst to best.

Survey responses are tested with the nonparametric binomial one sample one-tailed test. The sample value $X$ is computed using $z=((X-.5)-N P) / \sqrt{N P Q}$. For each survey question, the null hypothesis (H0) is that the "yes" (affirmative) response rate does not indicate a significant characteristic of video use. The alternative hypothesis (H1) is that the responses do identify a significant use. The "yes" response rates for rejecting the $\mathrm{H}_{0}$ are $67 \%$ at $\alpha=.01$ and $62 \%$ at $\alpha=.05$.

Observed course and final exam grades for student groups with and without the videos available $(\mathrm{N}=132$ and 33, respectively) are tested for significant differences. The nonparametric Kolmogorov-Smirnov large sample one-tailed test is used with $\alpha=.05$ and two degrees of freedom. The sample value $X=4 D^{2 *}\left(\left(n_{1} n_{2}\right) /\left(n_{1}+n_{2}\right)\right)$. The null hypothesis $\left(H_{0}\right)$ is that the grades were drawn from the same population and video lectures cause no significant grade improvement. The alternative hypothesis $\left(\mathrm{H}_{1}\right)$ is that video availability does significantly improve student grades.

\section{Video Mechanics}

Video lectures are feasible for the average non-IT instructor's use. Using a personal computer, an instructor can create them quickly and easily. They are not recordings of classroom lectures but cover lecture material as screen displays of content files with audio narrative (lecture) added. They can be produced before a course begins or developed as it progresses. The authors use both approaches, distributing videos for the entire course's coverage at the beginning of the semester, and then preparing a new video if needed to go over more slowly and extensively difficulties students are having with subject material. (A link to an example video used in this study can be found at http://www.csus.edu/indiv/b/brechthd/video/ch8.wmv)

Video lectures are Windows Media video files (wmv) created using Microsoft Producer as encoding software and Microsoft Office files for topic content. The encoding software captures screens from data files containing the materials used in classroom lectures and narrative audio from a microphone connected to the computer. They can be produced in the instructor's office or home, with no special set up required. Video lectures are more than technologically sophisticated lecture notes. Instead, they capture complete, instructor-quality instruction for all topics throughout a course.

In each video, the instructor navigates to display a topic-content file and delivers the audio lecture using the microphone. Chalkboard writing is simulated by using the keyboard and mouse pointer to write comments and highlight information on the screen. This development of topics has the feel of a live lecture, although no live classroom video or audio is edited into the video. In this study, the average time needed to produce a 75-minute video was about two hours, or four hours if there were retakes needed to correct mistakes. There was no incremental effort to create the Office files since they already existed for use in the classroom lectures.

The application of the Producer encoding software was beyond its recommended use with PowerPoint content files. Multiple types of files were used (PowerPoint, Excel, Word, and HTML - all created using Office software). Additionally, there was mouse pointer movement and keyboard writing, and screens were often scrolled. As a result, sections of a video could be difficult to watch with occasional blurred lines, "smudged" areas, or temporary flickering on the screen. The authors believe these problems are encoding issues with Producer that were encountered because of its unorthodox use. In a follow-up study using Microsoft Media Encoder, these difficulties were not encountered. Still, the Producer videos were viewable with enough clarity to be used by students without comment or complaint. 
The Appendix provides a more detailed discussion of video production mechanics. It covers software used; content files; screen writing/highlighting and narrative lecture; encoding configuration and techniques, and distribution to students.

\section{Study Population}

This study uses video lectures as a supplementary instructional method in a traditional, inresidence course. The course is a foundation, core course; is technically complex (accounting); and must be completed for students to progress to further studies in upper-division courses. Over ninety percent of the students pursue academic studies in areas other than the course's subject, and many have less than an optimal interest in or aptitude for the subject. The authors' university, one of twenty-three state universities in California, USA, is in a medium-sized metropolitan area. Students primarily live off campus and often work in part-time jobs.

Video lectures were introduced to this course to address this specific population of students and their interest and ability to learn this foundational subject. An area for further research that would extend the work of Hornik (Hornik, et. al, 2008) in a different direction is the application of video lectures to other lower division courses, technical and nontechnical, and to upper-division courses where students will have self-selected the subject of study and go into the course with a conceptual/methods foundation on which to build.

\section{Enabling a Teaching Strategy}

Video lectures make available instructor-quality lectures that students can view and study as much as needed to meet their individual learning needs. They are detailed step-by-step explanations of materials used in classroom lectures and are presented at a delivery pace that is significantly slower than what can be accomplished in the limited time available in the classroom. They can be paused and repeated and, thus, can be studied by students at their own learning pace.

Additionally, video lectures are a more focused learning experience than the traditional study of a textbook. Textbooks have a broad range of topics, and they develop topics in ways that may be inconsistent with the instructor's presentation of the material. Video lectures exclusively target what the student needs to learn, providing explanations the instructor is using in class. As discussed later, many students describe video lectures as a tutoring resource.

Additionally, video lectures allow the instructor to shift classroom time spent on basic, less challenging material to more complex and difficult subject material. By including more-complex information in classroom lectures, they are faster paced and provide the stimulation of more interesting material. Students who cannot fully understand and learn at this pace have the video lectures as a slower and very thorough second-lecture they can study at their own learning pace.

Finally, video lectures enable reducing classroom lecture time and substituting problem-solving labs, i.e., a change from lecture-based learning to active learning. In lab sessions, students are given a "practice problem" that they solve with open notes, alone or in groups, and with the instructor's one-on-one help when requested. The problems aggressively challenge and move forward students' understanding of and skill in working with the course material. After a lab, students can review an online solution of the problem, identifying and re-studying areas where they are incorrect or have weak understanding.

\section{Student Learning Preferences}

For video lectures to be effective, they must be accepted and used by students. They must provide an enjoyable or at least satisfactory learning experience, be perceived by students as providing a time-efficient study resource, and/or be perceived as improving understanding and grade per- 
formance. These factors involve, in part, the appeal of video lectures to individual learning preferences. While this relationship is an area for further research, some connection between video lectures and learning style preferences are commented on here.

In this study, students always have alternative study resources available - classroom lectures, the active-learning work done in the practice-problem labs, online lecture notes in the form of Office files, and the textbook. This availability of alternative resources supports the assumption that students' repeated use of video lectures evidences a learning preference for this form of study.

Education research has established that students have different ways in which they best learn new or difficult information (Dunn \& Dunn, 1993), and that it is important to address individual learning style preferences rather than attempt to instruct all learners with one style (Dunn, 2000; Dunn \& Griggs, 2000). The following factors, based on elements in the Dunn and Dunn Learning-Style Model (Dunn, 2000), illustrate learning preferences to which video lectures are expected to appeal. It is hypothesized that video lectures appeal to different learning styles from classroom lectures and may be an important, but not exclusive, determinant of their use.

1. Students may not learn most effectively from instructors' verbal explanations in a classroom lecture or from written materials (e.g., textbooks). Video lectures are expected to appeal to many students in the modern media culture, where the medium of information delivery may improve study effectiveness and learning.

2. In this study, video lectures are designed to appeal to an analytic learning style with more time spent on step-by-step explanations of concepts and problem-solving methods. In contrast, classroom lectures give an overview of the subject material and inherently appeal more to global learners. Students can enhance the analytic emphasis in video lectures by repeating step-by-step explanations.

3. A classroom lecture is presented to a large population of students, and it co-exists with a variety of sounds and other surrounding stimuli. Some students find this learning environment distracting and difficult. They may thrive when hearing the lecture in an environment they choose - a less distracting environment where they can better focus on lecture content. Also, students can study video lectures at a time of their choice, when they may be better able to concentrate and focus on the subject material.

4. A classroom setting typically imposes behavior and physical constraints on students. Video lectures may make better learning possible by enabling individuals to choose locations where they can take frequent breaks, move around, eat/drink, and enjoy physical comfort.

5. Finally, a student may have a learning preference for repeatedly studying one section of subject material until it is learned and then moving to the next section. Classroom lectures necessarily move through a large amount of material at one sitting. Video lectures allow pauses and repetition until sections of the material are learned.

\section{Student Acceptance and Use of Video Lectures}

The survey response rate for students with the videos available was $56 \%$ ( 74 out of 132 students). Students who reported using the videos were 73\% (54/74) of the responding students. The high use rate suggests that students broadly accept and use video lectures as a form of computer-based instruction and as an enhancement of traditional classroom courses.

The survey asked students how they used the video lectures and if they found them helpful in preparing for classroom lectures, understanding topics, doing homework, and preparing for exams. Following are the usage patterns reported. 
1. To understand better the concepts and problems presented in the classroom lectures. A surprisingly large percentage of students $(31.5 \%)$ viewed the videos in advance of classroom lectures. While this percentage is not statistically significant $(31.5 \%<62 \%$ for $\alpha=.05)$, its size contradicts a finding in the education literature that students study mostly in the two days prior to an exam, regardless of the education tools available (W. Maki and R. Maki, $2000)$. A statistically significant percentage of students $(68.5 \%>67 \%$ at $\alpha=.01)$ who used the videos - before or after the classroom lecture - said they helped understand the subject material.

2. To do homework. A statistically significant percentage of students $(72.2 \%>67 \%$ for $\alpha=$ $.01)$ used the videos to do homework. A statistically significant percentage of students $(66.7 \%>62 \%$ for $\alpha=.05)$ said the video lectures helped (5.5\% fewer students than used them). These results suggest student acceptance of computer-based instruction in learning to solve technically complex problems.

3. To prepare for weekly exams. A statistically significant percentage of students $(72.2 \%>$ $67 \%$ at $\alpha=.01)$ used the videos to prepare for weekly exams and found them helpful. Weekly exams are students' initial exams, and the process of studying for them reflects students' use of study resources for initial learning.

4. To prepare for midterm exams. The percentage of students who reported using the video lectures to prepare for midterm exams was not statistically significant $(61.1 \%<62 \%$ at $\alpha=$ $.05)$. However, a statistically significant percentage of students $(68.5 \%>67 \%$ for $\alpha=.01)$ report being helped by the videos for this purpose.

For many students, the process of studying for a midterm exam might be better characterized as review than initial learning. In this context, the use/helpfulness responses indicate the video lectures are often seen as useful for review, although a little less so than for initial learning (as seen in "1., 2., and 3. above").

The $61.1 \%$ of students who report using the videos to study for midterm exams is less than the $72.2 \%$ of students who used the videos to do homework and prepare for weekly exams. This decline is inconsistent with Maki \& Maki's (2000) finding that student use of study resources increases prior to an exam. An explanation is that video lectures are substantially appealing and effective for initial learning - obtaining general understanding, doing homework, and studying for weekly exams.

An explanation of the $7.4 \%$ difference $(68.5 \%$ - $61.1 \%)$ in students who were helped by the video versus those who used them to prepare for midterm exams is that some students did not use the videos as a study focus for midterm exams but either accessed them for a limited purpose or recalled the instruction they provided in prior use.

5. To obtain tutoring help. Finally, a statistically significant percentage of students $(63 \%>62 \%$ for $\alpha=.05)$ said the videos provided tutoring help. This response is remarkable compared to a lower positive response rate that might be expected if students were asked whether reading the textbook or class notes had tutoring value. Some possible tutoring qualities in video lectures are as follows.

(a) They have a focused-help quality in that $100 \%$ of the videos' coverage is relevant to what students must learn to succeed in the course. Students may view the videos as focusing on their needs. (b) The help provided by the videos is directly from the instructor, adding to a perception of the videos' focus, accuracy, and reliability in addressing learning needs. The explanations are also consistent with what they heard in the classroom lectures. (c) Students can pause or repeat segments of a video lecture, re-listening to the instructor's explanations 
until the material is understood. Students may experience this process as similar to repeatedly questioning a live tutor. (d) A sense of personal connection may come from listening to the instructor talk about the material and watching his or her comments being written on the computer screen. This continuing personal contact with the instructor removes some of the isolation of reading and studying written material.

\section{Student Learning/Grade Performance}

Responding to a survey question of whether the video lectures helped raise their course grade, $38.9 \%$ of all students using the videos said "yes". Statistically, this percentage is less than a critical value of $62 \%$ for $\alpha=.05$ and is not significant. Similarly, a statistical comparison of observed course grades for all students with videos available versus those without videos does not show statistically significant grade improvement (at $\alpha=.05$ ).

However, when grades for the two groups are graphed (Figure 1), it can be seen that with video lectures available, there are substantial differences in grades earned in the lower parts of the grade distribution, i.e., grades in the $0-50 \%$ and $50-60 \%$, and $60-70 \%$ ranges. When all students are included in the statistical analysis, this distinction is lost. Statistically, the grade improvement in the lower-end of the distribution is offset by a reversal of effect for the $70-80 \%$ and $80-90 \%$ grades and the closeness of the number of grades in the $90-100 \%$ range.

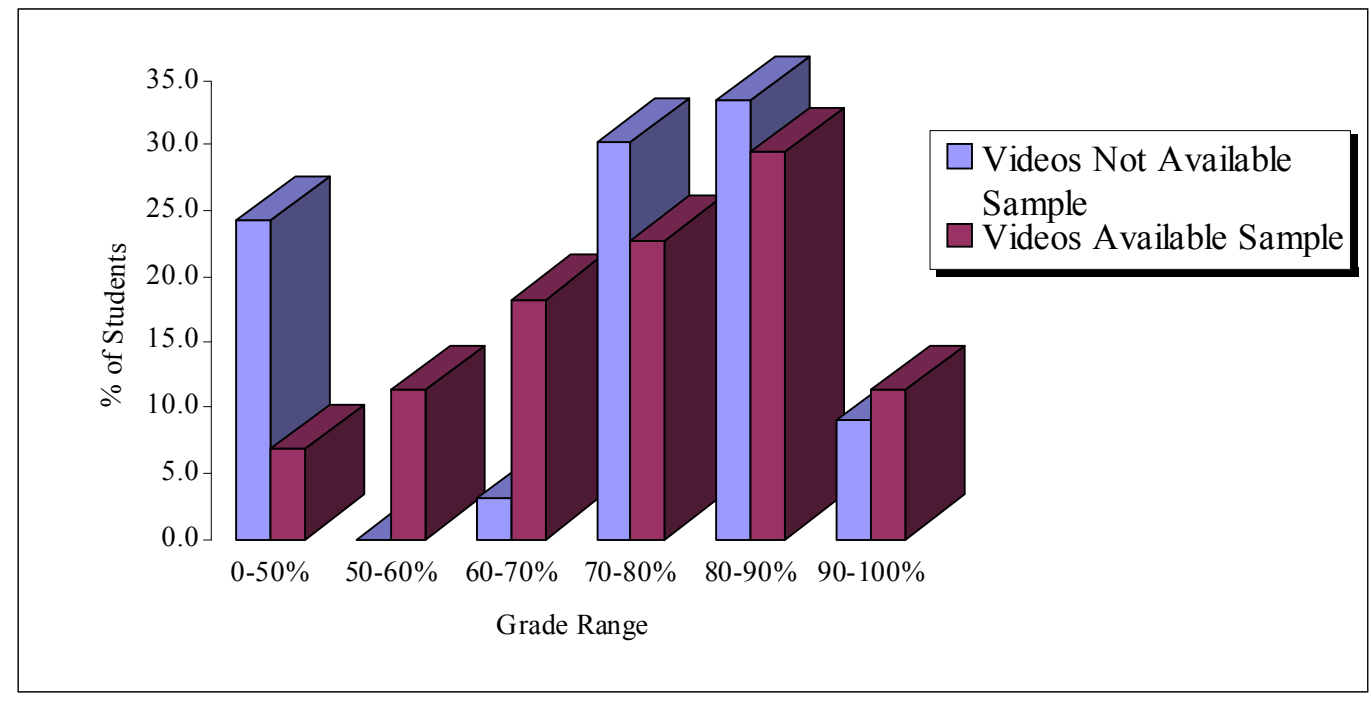

Figure 1: Course Grade Distributions

Addressing the extent to which video lectures help students in the lower end of the grade distribution, i.e., underachieving students, the survey asked all students if using the video lectures helped pass the class. $20.4 \%(11$ of $\mathrm{N}=54)$ said "yes". Qualitatively, this percentage is significant in terms of the educational goals that students learn and that they pass a required core course. Enabling students to learn is particularly important at a middle-level State University with a teaching mission that includes nontrivial numbers of students with substantially diverse motivations and capabilities.

Additionally, of the sample group without videos, $24.2 \%$ of the students are in the $0-50 \%$ grade range and failed the course. With the videos available, $6.8 \%$ of students are in this range. Subtracting, there are $17.4 \%$ fewer grades in the $0-50 \%$ range with videos available. This is a $71.9 \%$ $(17.4 \% / 24.2 \%)$ pass-rate improvement with videos available. 
A final learning-effects consideration is whether the availability of video lectures is associated with improved final exam grades. That is, does the evidence suggest that video lecture help students successfully review an entire semester's lectures in preparation for a comprehensive final exam? The available data are limited for this analysis because of survey anonymity and having conducted the survey before the final. More evidence is a direction for future research.

Nevertheless, if students' use of the videos to prepare for midterm exams and the underlying characteristics of this use are the same for the final exam, a tentative evaluation can be made. Figure 2 shows the distribution of final exam grades for students with and without video lectures available. The availability of video lectures is associated with students making fewer low grades in all of the $50 \%-70 \%$ grade ranges and more high grades in all of the $70 \%-100 \%$ ranges than if they did not have the videos. However, these improvements are not statistically significant (at $\alpha=.05)$.

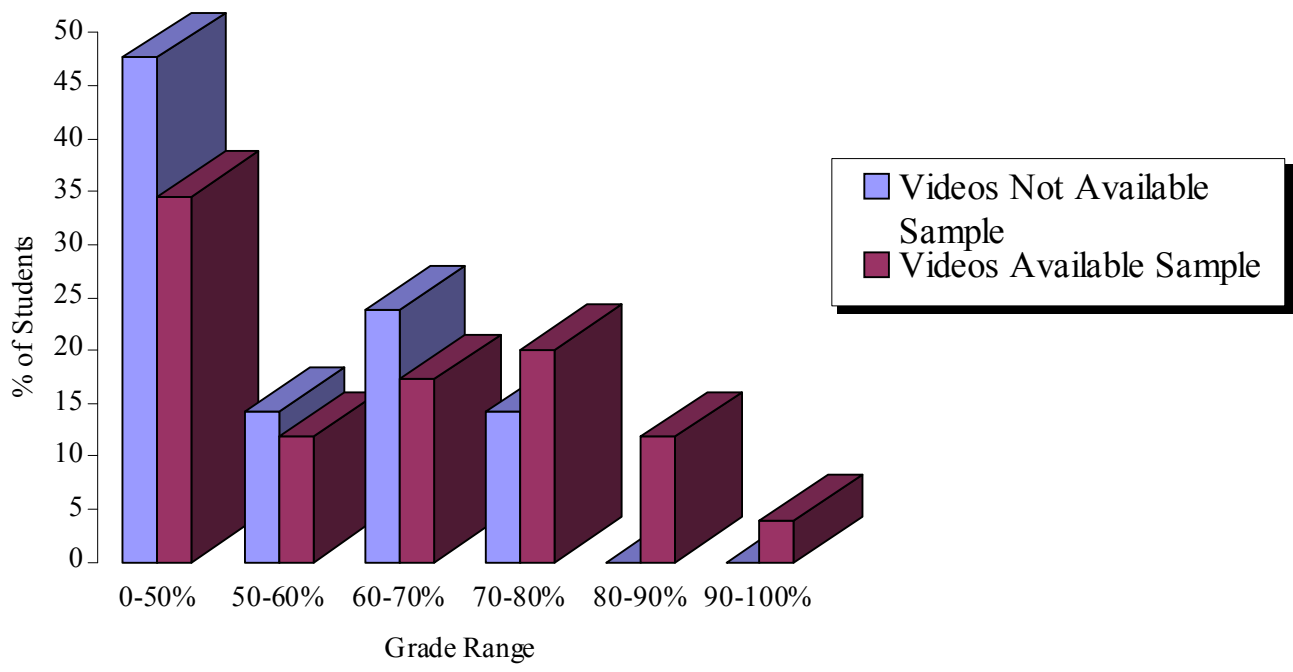

Figure 2: Comprehensive Final Exam Grades

Qualitatively, though, these grade improvements are important. An element of the teaching strategy, particularly for weaker students, is to offer the final exam as a motivating force to get students to learn the material even if late in the semester. The final exam grade replaces lower grades that were received during the semester for homework, practice problems, and exams - up to a maximum number. For example, a 74\% final exam grade replaces up to four homework and practice problem grades and up to three exam grades, but only for previous grades lower than $74 \%$.

To motivate students to attempt and accomplish improved performance, i.e., to attempt a last effort to learn the course material, they must believe that they can study all of the course's material and succeed on the final exam. Study resources are a key to this success, where instruction has ended and the final exam's content is comprehensive and as rigorous as that in the midterm exams. Video lectures support end-of-semester study by making an entire semester's lectures, delivered by the instructor, available. 


\section{Conclusion}

The education literature has noted that the learning effectiveness of many technology applications is unknown (Maki \& Maki, 2000). This study develops video lectures and addresses this deficiency for them. It is a study of video instruction's use as part of a teaching strategy. Student acceptance of video instruction, student use patterns, and videos' effects on learning are examined in a non-IT, traditional classroom course. Qualitative and nonparametric statistical analyses are used to examine survey responses from students with the videos available and grades from students with and without the videos available.

Video lectures are web and CD viewable video files, created in the instructor's office with free and easy to use media file encoding software. They are a study resource that provides classroom lecture content but are more than technologically sophisticated lecture notes or recordings of classroom lectures. They are produced using Microsoft Producer software for encoding, Microsoft Office files for topic content, and a microphone for the instructor's audio lecture. Chalkboard writing is simulated by using the keyboard and mouse pointer, giving the lectures the look and feel of a live lecture. For this study, the time needed to produce a 75-minute video was about two hours where the Office files already existed for use in the corresponding classroom lectures.

This study is experimental in testing whether video lectures enable a teaching strategy used throughout a course. The videos repeat the course instructor's classroom instruction, with the video lectures presented at a slower, more detailed, and more thorough pace. They are relied upon to meet weaker students' extended teaching needs at a university with a mission of teaching a broad spectrum of students. They free classroom time so that more challenging and complex coverage can be added to classroom lectures. Additionally, they enable classroom lecture time to be reduced and converted to problem-solving labs that emphasize active learning.

In the lab sessions, students are given complex and methods-comprehensive problems to solve, where they can use notes, work in groups, and call on the instructor for help. The problems aggressively challenge and move forward students' skill in understanding concepts and working with the course material.

For video lectures to be most effectively used by students, they should appeal to students learning style preferences. Individual preferences may differ from those appealed to by classroom lectures and written resources such as textbooks or course notes. Video lecture appeals are as follows: (a) Their content is $100 \%$ relevant to course performance requirements and is presented at a more detailed pace than classroom lectures; (b) Videos can be replayed and enable students to repeat the instructor's explanations; (c) They can be viewed at a time, location, and under environmental conditions of a student's choice; (d) Their portability enables listening and study without the competing distractions that often accompany classroom lectures.

Students use the video lectures to (a) understand concepts and problems presented in the classroom lectures, (b) do homework, (c) prepare for weekly exams, and (d) receive instructor-quality tutoring assistance. The number of students using and being helped by the videos for these purposes was statistically significant in all cases. Large but statistically insignificant numbers of students used the videos to prepare before classroom lectures $(31.5 \%)$ and for midterm exams $(61.1 \%)$.

All of these uses indicate broad acceptance of computer-based instruction from the course instructor in a course that is not advertised as computer enhanced. They indicate that video lectures are substantially appealing to many students and are perceived as effective for learning. As a percentage of the student population responding to the survey and using the videos, video lectures help large numbers of students raise their course grades $(38.9 \%)$. 
Comparing the average percentage of students without videos who fail the course with the percentage who failed with the videos available, there is a $71.9 \%$ reduction in failing grades with the videos available. These data indicate that video lectures help achieve important educational goals of learning improvement and retention for students most at risk of failure.

Finally, video lectures make an entire semester's lectures available for study at the end of the semester in preparation for the final exam. Analysis of students' comprehensive final exam grades shows improvement in all grade ranges with videos available. This improved last-minute learning was expected, although the results are not statistically significant in terms of the number of students benefited.

An appealing extension of video instruction is to online courses. However, as the term "video lectures" is used in this study, it refers to the use of video instruction to support a comprehensive teaching strategy. This strategy enables improved performance for weaker students, a stronger curriculum, and more classroom time spent on active learning. In addition, video lectures are used as supplements to classroom lectures, and there is ongoing instructor-student interaction that may contribute to making video lectures effective, particularly in having tutoring value. A direction for future research is to investigate how video instruction may strengthen or broaden teaching strategies that are possible in online education, and how their use in online courses may simulate the teaching dynamics of in-residence courses.

An application of video instruction that is particularly appealing to this study's authors is to use short-summary instructional videos in an online tutoring library for students. This application would have many of the properties of videos discussed in this study and by Whatley and Ahmad (2007). A college's budget resources for live tutors are limited, and student-tutors' competence may be limited to their previous course experience and personal capabilities. An online library of video tutorials that is available to all students in an academic area could greatly expand free tutoring availability and extend it to many students who would not seek out a live tutor. These videos would have the competence of being taught by regular faculty instructors.

\section{References}

Cuban, L. (2001). Oversold and underused: Computers in the classroom. Cambridge, MA: Harvard University Press.

Dunn, R. (2000). Capitalizing on college students' learning styles: Theory, practice, and research. In R. Dunn \& S. Griggs (Eds.), Practical approaches to using learning styles in higher education (p. 10). Westport, CT: Praeger.

Dunn, R., \& Dunn, K. (1993). Teaching secondary students through their individual learning styles: Practical approaches for grades (pp. 7-12). Boston: Allyn \& Bacon.

Dunn, R., \& Griggs, S. (2000). Practical approaches to using learning styles in higher education: The howto steps. In R. Dunn \& S. Griggs (Eds.), Practical approaches to using learning styles in higher education (p. 29). Westport, CT: Praeger.

Hornik, S., Saunders, C., Li, Y., Moskal, P., \& Dzuiban, C. (2008). The impact of paradigm development and course level on performance in technology-mediated learning environments. Informing Science: The International Journal of an Emerging Transdiscipline, 11, 35-58. Retrieved from http://inform.nu/Articles/Vol11/ISJv11p035-058Hornik121.pdf

Keengwe, J. (2007). Faculty integration of technology into instruction and students' perceptions of computer technology to improve student learning. The Journal of Information Technology Education, 6 , 169-180. Retrieved from http://jite.org/documents/Vol6/JITEv6p169-180Keengwe218.pdf

Maki, W. \& Maki, R. (2000). Evaluation of a web-based introductory psychology course: II. Contingency Management to Increase Use of On-Line Study Aids, Behavior Research Methods, Instruments and Computers, 2(32), 240-245. 
Sarker, S. \& Nicholson, J. (2005). Exploring the myths about online education in information systems. Informing Science: The International Journal of an Emerging Transdiscipline, 8, 55-73. Retrieved from http://inform.nu/Articles/Vol8/v8p055-073Sarker.pdf

Whatley, J. \& Ahmad, A. (2007). Using video to record summary lectures to aid students' revision. Interdisciplinary Journal of E-Learning and Learning Objects, 3, 185-196. Retrieved from http://ijello.org/Volume3/IJKLOv3p185-196Whatley367.pdf

\section{Appendix: Detailed Discussion of Video Production Mechanics}

The discussion in this appendix reflects experience gained in the production of videos lectures for this study and subsequently.

\section{Software Used}

All of the software used was and continues to be from Microsoft. Producer was used for this study to encode the videos; however, Encoder 9 Series has been used since this study. Producer and Encoder create Windows Media video files (wmv files). Wmv files play in either Windows Media Player or RealPlayer, so their use is not an issue for distribution and student use. Microsoft Office files are used for topic content, including PowerPoint, Excel, Word, and HTM files.

A partial reason for using Microsoft software is its apparent universality. Given a large number of students that a university registers over time, students commonly have familiarity with Microsoft products, at least at the authors' university in the USA. Even Mac computers have software that makes them able to run Microsoft Office and wmv files.

More importantly, the choice of Microsoft products is grounded in Office being the information processing/creation standard for the authors' College and its Management Information Systems academic department. Students possess and are trained in the use of PowerPoint, Excel, and Word software, which is also available in campus labs. Thus, students are able to use video lecture content files that are made available for download through the course web site and a CD that is now distributed to students at the start of each semester.

Producer was chosen for this study as the encoding software because Microsoft was marketing it as a solution for the type of application the authors envisioned for video lectures. It was the authors' initial effort in encoding video lectures, and, incidentally, Producer was a free download at the time of the study. After developing the videos for this study and realizing the encoding difficulties encountered (as discussed in the body of the paper), a phone call to Microsoft led to the suggestion to use Encoder. Encoder is a free download from the Microsoft link http://www.microsoft.com/windows/windowsmedia/forpros/encoder/default.mspx

Rather than using Microsoft encoding software, alternative software may be desirable for a developer, for example, RealNetworks' RealProducer or an Adobe Flash-Media encoding product. Some IT-professionals in the authors' university IT-group advised the use of RealNetworks' encoding software. However, the use of Office content files led the authors to initially chose Producer and to now use Encoder. The authors see Microsoft encoding software as a best bet for timely stability and effectiveness in encoding performance of Microsoft Office content files across time, where the code in the content files evolves/changes in new Office versions. 


\section{Content Files}

For the authors, the greatest use of time is in creating the content files, not in recording a video lecture or giving the narrative instruction. Issues concern the type and structure of content files.

The authors have experimented with using different types of files - from the mix of files used in this study (PowerPoint, Word, Excel, and HTM), to solely using PowerPoint slides, to solely using Word files (the authors' current preference). Goals are to display the content being lectured with maximum content per screen and to move through a series of screens easily and smoothly without interrupting the discussion.

What the authors view as major difficulties with PowerPoint slides are having to cram a lot of information into the confines of a single slide and the sense of discontinuity created in a lecture by having to toggle between different slides to encompass the total content of a concept or problem.

Alternatively, topic development in a single Word file seems to flow seamlessly, with more information contained in a single screen than in a PowerPoint slide and a smooth movement through the material by scrolling down the minimum needed to display the next element of the discussion.

Additionally, with the capabilities of Word files, Excel files are unneeded if the sole purpose is to display finished content. For instance, material can be developed in Excel and pasted into Word. Excel is probably a better platform for content files if the instructional goal is to demonstrate the process of making a series of computations. And, of course, a particular software platform is appropriate if the goal is to demonstrate the configuration or use of that software. Finally, with Word, browser links can be accessed from within the file, and images can be pasted into Word files and resized or formatted as needed.

Regarding the structure of content files, the developer must decide how much time to spend putting lecture content in written form. Possibilities include the following.

1. Simply use whatever PowerPoint slides are distributed as instructor resources with an adopted textbook. Files with solutions for end-of-chapter assignments may also be provided as part of an instructor's textbook adoption package. If these files are available and used, there is essentially zero content development and file preparation time required. Files are downloaded from the textbook web site or copied from an instructor resource disk and become the content files for the video lectures.

2. Alternatively, the textbook resource files (in "1" above) may be enhanced to include material unique to the course as taught. This approach to content file development is still time efficient because existing files are only being customized.

3. A third approach to creating content files is to build them from the ground up to fit the unique content and presentation style used in the course. This approach can be relatively simple or elaborate, with corresponding implications for the development/production time required.

The authors have used all three approaches as they build and change content files as a course is developed and improved. While video lectures must be redone as content files change, a video lecture is prepared in an hour or two if the instructor is simply recording known lecture content with existing content files. 


\section{Screen Writing/highlighting \& Narrative Lecture}

In the classroom, with an image projected to the side of the chalkboard by the computer, an instructor verbally adds lecture and written comments or computations on the chalkboard. The same thing occurs in video lectures, where the keyboard or mouse is used to enter the chalkboard writing. Content entry using the keyboard is easier to do when Word software (or Excel) is displaying the content file, as opposed to PowerPoint where slide space and structure limitations can make ad hoc content entry very problematic. Using Word, content is easily added, as it would be in any word-processing action.

Concerning the playing length of the video, the authors have moved toward writing much less and having more prepared material already in the content file. A key objective is to shorten the playing time in order to avoid student loss of interest. Excessive writing lengthens playing time. Exploiting highlighting by the mouse is an alternative and quick way to focus attention on a section of content and still retain the sense of the instructor connecting his or her words to a chalkboardtype action.

For the audio narration, the instructor uses a microphone attached to the computer. Lecturing this way is the same as lecturing in the classroom. It is convenient to have a microphone headset so that the instructor's attention in giving the lecture is focused on the screen displays and navigation through the screens. The authors have found that investing about $\$ 50$ (US dollars) in a highquality headset is more comfortable to use and gives better audio quality than the cheap microphones that come with computers. The headset the authors use is marketed for use with computer games.

\section{Encoding Configuration and Techniques}

The computer should have sufficient RAM to keep open the encoding software, whatever Office products are used to open files, and all the content files that will be used. Having all of these programs and files open speeds navigation between content files and shortens video playing time.

All the instructor has to do to record a video lecture is start Encoder and then navigate to content files, talk, and highlight/write into the information processing file that is on display on the screen. When through with the lecture, stop Encoder and save the wmv file. Using the $<$ alt $><\operatorname{tab}>$ keyboard combination to toggle between programs provides fast navigation. Within Word 2003, the "previous window"/"next window" menu buttons can be clicked to navigate, with the buttons placed on a toolbar.

The authors do not attempt to crop the beginning display when Encoder is started or the ending display when the instructor toggles back to Encoder and clicks the Stop button. Additionally, no editing software is used to correct mistakes or otherwise enhance a video. A downside to this approach is the need to re-record the entire lecture if a major mistake is made. Otherwise, the result is a little imperfect, but overall it requires much less video production time.

Learning how to set up and repeatedly use an Encoder configuration may require technical assistance. There are several parameters to consider, and configuring, saving, and initiating a template may require initial help. Encoder does have complete help references.

A core configuration issue is to maintain video and audio quality while limiting the size of a wmv file. Large wmv files are space consuming and slow to upload to university servers. Wmv files created with highest quality video and lossless quality audio settings are so large that a single 75minute file can consume a majority of a 700 megabyte $\mathrm{CD}$ and be completely infeasible for remote upload to a server. 
At the authors' university, server capacity is freely available to faculty, with media files placed on the regular course web site server. Although free, it is understood that server space is a scarce resource, and file size is an issue. For the sixteen wmv files available to students in this study, the most common file size was about 17 megabytes, with files ranging from 10 to 32 megabytes in size. Using Producer, a total of 237 megabytes were used.

Currently, the Encoder video and audio quality settings the authors use are medium quality as shown in Figure 3. The video/audio quality is better than in this study's files, but file size has increased to mostly 28 to 35 megabytes per file.

Sources Output Compression Video Size Altibutes Processing Plug-ins Security Advanced
Select the distribution method and encoding settings for your content. The distribution method you
select determines the seltings that are available.
Destination: File archive
Video: Medium quality video NBR 75]
Audio: Medium quality audio (NBR 50]

Figure 3: Encoder Quality Settings Currently Used

\section{Distribution to Students}

At the time of this study, the videos were available to students solely through the class web site and could be viewed online either at home or at the university's computer labs. However, home viewing of 75-minute videos is infeasible for students with dial-up connections, and the cost of high-speed connections makes this access unavailable to the poorest students.

Today, the pervasive availability of DSL, cable, and high-speed satellite connections in the USA makes home online access relatively inexpensive and readily available. Even so, CDs containing the videos are distributed at the start of the semester as a way to eliminate cost as a consideration for access and to ease and promote the videos' use.

\section{Biographies}

H. David Brecht is a Professor of Accounting. His principal areas of interest have varied over the years - from managerial accounting with emphases on behavioral/organizational dynamics and computer information systems, to auditing, accounting information systems, and currently pedagogy in the use of information technology in teaching financial accounting. He has published in academic and professional journals, including decision sciences and the above accounting areas of interest.

Suzanne M. Ogilby is the Associate Dean of Faculty and a Professor of Accounting. Her principal areas of interest include financial, governmental, and nonprofit accounting, including innovative pedagogy in teaching these areas and topics that pose particularly difficult learning challenges to students. She has published in academic and professional journals including the above areas of interest. 